examination cannot comprehensively assess the molecular heterogeneity in the tumour microenvironment. We aim to capture tissue morphology and single cell protein expression of cancer and immune cells within intact tissue sections of stage III colorectal tumours from 11 patients.

Methods: Using Hyperion Image Mass Cytometry (IMC), we simultaneously profiled 16 protein markers from FFPE samples, including cancer (Ki-67, p53) and immune (CD3, CD4, CD8a, CD45RO, CD20, CD68, Granzyme B) cell markers. We integrated this with matched histopathological imaging and wholeexome-sequencing data.

Results: We identified seven cancer, immune and stromal cell types and preserved their spatial organisation within the microenvironment. These cell types were validated on matched $\mathrm{H} \& \mathrm{E}$ histopathology. We found significant interactions of cancer cells with NK-cells and B-cells. Furthermore, we found that tumours with $17 p$ deletion had significantly fewer NK-cells.

Conclusion: Using IMC to acquire rich spatial data at single-cell resolution enabled us to assess the heterogeneity of tumour tissue by defining cell types, immune infiltration, and cancer-immune cell interaction within intact tissue sections. Integration of this data with $H \& E$ images could assist pathologists in cancer diagnosis.

\section{GASTRIC EPITHELIAL ATYPIA ASSOCIATED WITH RADIATION}

Stefi $^{1}$, Abha Malik ${ }^{2}$, Bryn Atmore

${ }^{1}$ Department of Anatomical Pathology, Australian Clinical Labs, Bella Vista, NSW, Australia; and ${ }^{2}$ Department of Anatomical Pathology, Australian Clinical Labs, Northern Beaches Hospital, NSW, Australia

\begin{abstract}
Aim: Radiotherapy is widely accepted treatment in advanced cancer with a high risk of local recurrence. Radiation induced cancers are well described, however less attention has been given to non-neoplastic mucosa. Our aim is to provide a detailed analysis of morphological features present in non-neoplastic mucosa in order to avoid misdiagnosis.

Method: We reviewed the case of 74 years man with a history of unknown primary and documented bony metastasis, presented with gastric ulcer, for which histological examination along with immune-histochemical (IHC) analysis was performed.

Results: Histology, mucosal ulceration, acute inflammation, eosinophilic infiltrates and architectural abnormalities. Epithelium atypia had a lower nuclear: cytoplasmic ratio, aiding distinction from dysplasia. IHC for cytokeratin and viral inclusions was done. Further work-up on the case revealed that the medial part of the stomach was in the radiation field, which helped to reach the final diagnosis of radiotherapy induced atypia.

Discussion: The gastrointestinal (GI) tract, particularly the bowel, is very vulnerable to radiation damage because of the anatomical location and the high cell turnover. Radiation induced changes resemble dysplasia, viral cytopathic and in chronic cases many resemble IBD. Confident diagnosis can be made in conjunction with detailed history, histology and IHC.

\section{'FIJI BELLY' CULMINATING IN COLECTOMY: A CASE REPORT FROM NEW ZEALAND WITH BRIEF REVIEW OF LITERATURE}

$\underline{\text { Paola T. Bravo }}{ }^{1}$, Fouzia Ziad $^{1}$, Simione Lolohea ${ }^{2}$
${ }^{1}$ Department of Anatomical Pathology and Cytology, Waikato Hospital, New Zealand; and ${ }^{2}$ Department of Surgery, Waikato Hospital, New Zealand

Traveller's diarrhoea due to Entamoeba histolytica is a worldwide problem due to growing popularity of international travel to exotic locations. Acute fulminant or necrotising amoebic colitis is a rare but potentially fatal complication of intestinal amebiasis, reported in only less than $0.5 \%$ of cases.

We report the case of a 55-year-old male who became unwell after travelling to a rural area in Fiji. He was initially treated as diverticulitis and subsequently had appendectomy due to suspected appendicitis. Symptoms persisted and subsequent colonic biopsies revealed features of acute colitis and revealed no microorganisms. Patient was treated for inflammatory bowel disease with Pentasa and prednisone, but continued to deteriorate. Fulminant colitis was suspected based on imaging findings and a subtotal colectomy was performed. Histopathological examination revealed flask shaped ulceration and areas of necrosis containing abundant microorganisms morphologically consistent with Entamoeba. Patient recovered well after antiamebic medication.

\section{GASTRITIS CYSTICA POLYPOSA IN AN UNOPERATED STOMACH, A CASE REPORT}

\author{
Kai Zong Teo, Nazmoon Laila \\ Department of Anatomical Pathology, Australian Clinical Labs, \\ Bella Vista, NSW, Australia
}

Gastritis cystica polyposis (GCP) is a rare benign hyperplastic polypoid neoplasm that usually occurs on the gastric side of anastomoses in patients who have previously undergone gastroenterostomy surgery. Only few cases of GCP have been reported in a previously unoperated stomach. Here we present a 73-year-old female who on investigation for altered bowel habits was found to have a gastric polyp diagnosed as GCP. Both endoscopic and radiological findings are non-specific, and diagnosis depends on histopathological evaluation of the lesion. Histologically the lesion showed a hyperplastic polyp with cystically dilated foveolar and glandular epithelium extending into the submucosa surrounded by disorganised bundles of smooth muscle and without any evidence of cytologic atypia, typical of GCP. Although benign, an associated with gastric carcinoma has been described.

\section{SEX CORD TUMOUR WITH ANNULAR TUBULES}

Amitha Thomas, Mila Volchek

Anatomical Pathology Department, The Royal Women's Hospital, Parkville, Vic, Australia

Sex cord tumours with annular tubules are a rare but distinct variant of sex cord stromal tumours, first described by Scully in $1970 .^{1}$ A third of cases are found in patients with Peutz- Jeghers syndrome (PJS) whilst the remaining arise sporadically. ${ }^{2}$ Sporadic SCTATs are unilateral, larger and not calcified as opposed to PJS associated lesions which are multiple, small, bilateral and calcified. ${ }^{1,3}$ Sporadic tumours have a greater metastatic potential than their syndromic counterpart. Histologically, sporadic lesions show more differentiation into Granulosa and Sertoli cell 
tumour. $^{3}$ This case study reports a patient with all the characteristic features of a sporadically arising tumour.

References

1. Scully RE. Sex cord tumour with annular tubules a distinctive ovarian tumour of the Peutz-Jeghers syndrome. Cancer 1970; 25: 1107-21.

2. Young RH, Welch WR, Dickersin GR, et al. Ovarian sex cord tumor with annular tubules: review of 74 cases including 27 with Peutz-Jeghers syndrome and four with adenoma malignum of the cervix. Cancer 1982; 50: 1384-402.

3. Bennett P, Williamson C. Basic Science in Obstetrics and Gynaecology. 4th ed. London: Churchill Livingstone, 2010.

\section{GASTRIC SCHWANNOMA: A RARE BUT IMPORTANT DIFFERENTIAL FOR GIST}

Amitha Thomas, Ju Lee H'ng

Anatomical Pathology Department, Eastern Health, Box Hill

Hospital, Box Hill, Vic, Australia

Gastric schwannomas are a rare mesenchymal tumour. They are often detected incidentally and present with nonspecific symptoms. ${ }^{1}$ They can be mistakenly diagnosed as a gastrointestinal stromal tumour (GIST) preoperatively. We present a case of a 26-year-old male with a presumptive preoperative diagnosis of a GIST that was confirmed to be a gastric schwannoma on histology.

\section{References}

1. Shah AS, Rathi PM, Somani VS, et al. Gastric schwannoma: a benign tumor often misdiagnosed as gastrointestinal stromal tumor. Clin Pract 2015; 5: 775.

\section{MULTIPLEXED IMMUNOFLUORESCENCE IDENTIFIES STROMAL CD68 ${ }^{+}$PD-L1 ${ }^{+}$MACROPHAGES AS A PREDICTOR OF OUTCOME IN TRIPLE NEGATIVE BREAST CANCER}

James Wang $^{1}$, Lois Browne ${ }^{2}$, Iveta Slapetova ${ }^{3}$, Fei Shang ${ }^{3}$, Kirsty Lee $^{4}$, Jodi Lynch ${ }^{2}$, Julia Beretov ${ }^{1,2,5}$, Renee Whan ${ }^{3}$, Peter Graham ${ }^{1,2}$, Ewan KA. Millar ${ }^{1,5,6,7}$

${ }^{1}$ St George \& Sutherland Clinical School, University of New South Wales Sydney, Kensington, NSW, Australia; ${ }^{2}$ Cancer Care Centre, St George Hospital, Kogarah, NSW, Australia; ${ }^{3}$ Biomedical Imaging Facility, Mark Wainwright Analytical Centre, University of New South Wales Sydney, Kensington, NSW, Australia; ${ }^{4}$ Department of Clinical Oncology, Prince of Wales Hospital, Chinese University of Hong Kong, Shatin, Hong Kong; ${ }^{5}$ Department of Anatomical Pathology, New South Wales Health Pathology, St George Hospital, Kogarah, NSW, Australia; ${ }^{6}$ Faculty of Medicine \& Health Sciences, Western Sydney University, Campbelltown, NSW, Australia; and ${ }^{7}$ University of Technology, Sydney, NSW, Australia

Background: Triple negative breast cancer (TNBC) comprises $10-15 \%$ of all breast cancers and has a poor prognosis. Our aim was to characterise the immunophenotypes of stromal immune cells expressing PD-L1 and determine their association with overall survival (OS) and breast cancer-specific survival (BCSS).
Methods: Tissue microarrays were constructed from a retrospective cohort of TNBC patients from St George Hospital $(n=244)$. Multiplexed immunofluorescence staining for CD3, CD8, CD20, CD68, PD-1, PD-L1, FoxP3 and pan-cytokeratin was performed using the Vectra Polaris ${ }^{\mathrm{TM}}$ and analysed using QuPath. Cox univariate and multivariate analyses (MVA) determined associations with outcome using the median cell count as the cut-point.

Results: In MVA analyses, high $\mathrm{CD} 68^{+} \mathrm{PD}-\mathrm{L}^{+}{ }^{+}$was associated with improved prognosis for OS $(\mathrm{HR}=0.56,95 \%$ CI $0.33-0.95$, $p=0.030)$ and BCSS (HR=0.47, 95\% CI 0.25-0.88, $p=0.018)$ in the whole cohort and in patients receiving chemotherapy $(n=174$; OS HR $=0.43,95 \%$ CI $0.22-0.83, p=0.012$; BCSS HR $=0.38$, $95 \%$ CI $0.18-0.78, p=0.009$ ), improving on the predictive value of PD-L1 ${ }^{+}$alone for BCSS.

Discussion: High $\mathrm{CD} 68^{+} \mathrm{PD}-\mathrm{L1}^{+}$macrophages define a group of TNBC patients with improved prognosis. Our data supports the recent description of $\mathrm{CD}^{+} 8^{+} \mathrm{PD}-\mathrm{L1}^{+}$macrophages predicting response to neoadjuvant immunotherapy in $\mathrm{TNBC}^{1}$ and its potential as a more specific immune biomarker.

Reference

1. Ahmed FS, Gaule P, McGuire J, et al. PD-L1 protein expression on both tumor cells and macrophages are associated with response to neoadjuvant durvalumab with chemotherapy in triple-negative breast cancer. Clin Cancer Res 2020; 26: 5456-61.

\section{RETE CYSTADENOMA OF THE OVARY: A DISTINCTIVE AND POSSIBLY UNDER-REPORTED ENTITY}

Anders S. Wong, Marsali Newman, Alison Skene

Department of Anatomical Pathology, Austin Hospital, Melbourne, Vic, Australia

Rete cystadenoma is an uncommon neoplasm with distinctive morphological features, including a characteristic epithelial lining, and prominent smooth muscle bundles and hyperplastic hilus cells within the wall. It has limited exposure in the literature and is probably underdiagnosed. Whilst both rete cystadenoma and its more common mimic serous cystadenoma are benign, recognition of the former is important because of the association with systemic symptoms, in particular virilisation, which can occur in up to $50 \%$ of the cases. Here, we present a case of rete cystadenoma to draw attention to this distinctive entity, and demonstrate ER and PR negativity in the cyst wall epithelium, which may contrast to that of serous cystadenoma.

\section{MISSED OPPORTUNITIES FOR THE DIAGNOSIS OF HYPERCORTISOLISM: AUDIT OF TEST SELECTION PREFERENCES IN PRIMARY CARE}

Rachel J. M. Dennis, Johan H. Conradie, Melissa J. Gillett, Michael M. Page

Western Diagnostic Pathology, Jandakot, WA, Australia

Hypercortisolism is associated with significant morbidity and mortality. ${ }^{1}$ Recommended initial tests for hypercortisolism include urine free cortisol (UFC), late-night salivary cortisol 